\title{
A narrative review of the management of benign prostatic hyperplasia in patients undergoing penile prosthesis surgery
}

\author{
Lexiaochuan Wen^, Tobias S. Köhler, Sevann Helo^ \\ Department of Urology, Mayo Clinic, Rochester, MN, USA \\ Contributions: (I) Conception and design: All authors; (II) Administrative support: All authors; (III) Provision of study materials or patients: All \\ authors; (IV) Collection and assembly of data: All authors; (V) Data analysis and interpretation: All authors; (VI) Manuscript writing: All authors; (VII) \\ Final approval of manuscript: All authors. \\ Correspondence to: Lexiaochuan Wen, MD. 200 1st Street SW, Rochester, MN 55905, USA. Email: Wen.Lexiaochuan@mayo.edu.
}

\begin{abstract}
Penile prosthesis surgery is an effective and durable treatment modality for patients who have failed conservative management for erectile dysfunction (ED). Thorough patient counseling and appropriate preoperative workup lay the foundation for a successful outcome. While the risk of infection of penile prosthesis is rare, it is a dreaded complication with dire consequences. The goal of the prosthetic surgeon is to minimize the risk of preventable complications. Given the common prevalence of benign prostatic hyperplasia $(\mathrm{BPH})$ in this patient population, it is essential that providers are familiar with the implications and nuances of managing both conditions in order to maximize the chances of a favorable result. Due to the relatively infrequent nature of complications associated with the management of BPH in the setting of a penile prosthesis, literature regarding this topic is scarce. In this narrative review we present our own case series illustrating some of the most common scenarios that a prosthetic surgeon may encounter. We have included our suggestions for management in these difficult situations based on our clinical experience. In the following review we have highlighted the importance of identifying and treating BPH in penile implant candidates to reduce postoperative morbidity and to offer critical insights into managing BPH-related complications this population.
\end{abstract}

Keywords: Lower urinary tract symptoms (LUTS); prostatic hyperplasia; penile prosthesis; penile implantation; urinary bladder neck obstruction

Submitted Aug 31, 2020. Accepted for publication Jan 13, 2021.

doi: $10.21037 /$ tau-20-1225

View this article at: http://dx.doi.org/10.21037/tau-20-1225

\section{Introduction}

Penile prosthesis surgery (PPS) has revolutionized management of medication-refractory erectile dysfunction (ED) and greatly improved the lives of patients affected by this condition. For patients who have failed first-line therapy such as phosphodiesterase- 5 inhibitors, vacuum erection device, or intracavernosal injections, penile prosthesis provides dependable and durable results. The most commonly implanted prosthetic device is the inflatable penile prosthesis (IPP), which boasts the highest patient and partner satisfaction rates, $92 \%$ and $95 \%$ respectively (1-3). Patient selection, preoperative counseling, and management of patient expectations are critical for surgical success and patient satisfaction. Understanding common comorbid conditions in this patient population not only helps define management algorithms but also prepares surgeons to anticipate perioperative challenges.

Lower urinary tract symptoms (LUTS) due to benign prostatic hyperplasia (BPH) is a common comorbid

^ ORCID: Lexiaochuan Wen, 0000-0001-8912-3899; Sevann Helo, 0000-0002-3274-3672. 


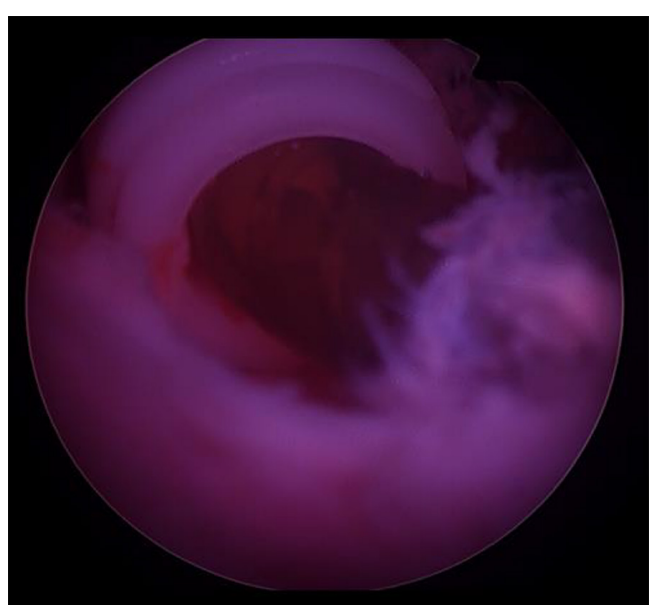

Figure 1 Implant tubing seen in urethra during PVP procedure.

condition in men with ED, and is present in up to $72 \%$ of men with ED (4-7). The degree of ED correlates with LUTS severity, with men with more severe LUTS symptoms reporting lower sexual satisfaction and decreased libido (8). While both patient populations share common risk factors, large scale cross-sectional studies have shown the correlation exists even after accounting for cofounding variables such as age and vascular risk factors $(9,10)$. Despite these correlative studies, we have yet to uncover the shared mechanism between the two conditions. Current understanding suggests that dysfunctions in smooth muscle contractility, metabolism, sex hormones, and inflammation may all play a role in the pathogenesis of both (11). The preponderance of LUTS/BPH in patients undergoing consideration for IPP has important management implications that will be discussed in this review including preoperative workup, surgical considerations, and postoperative challenges of managing $\mathrm{BPH}$ in patients undergoing PPS. Management of LUTS/BPH in the setting of PPS represents a gap in the current surgical literature which we seek to add to with this review of the existing literature and discussion of our clinical experience. We present the following article in accordance with the Narrative Review reporting checklist (available at http:// dx.doi.org/10.21037/tau-20-1225).

\section{Case series of penile prosthesis infections after urinary tract instrumentation}

We would argue that urinary tract instrumentation after PPS poses a clear infection risk to the prosthetic device despite the absence of supporting literature. The absence of cases in the literature should not be interpreted as evidence for safety, but rather a reflection of the infrequent nature of such a complication that may go unreported, and is difficult to prove. Given the absence of cases in the literature regarding injury or infection of a penile prosthesis device due to urethral instrumentation, we present our own case series to illustrate this topic.

\section{Case 1: uretbral injury from bladder outlet obstruction procedure after PPS}

A 64-year-old man who previously underwent insertion of a three-piece IPP device 1 year prior subsequently developed LUTS. He was referred for water vapor thermal therapy. The patient was evaluated, but was not felt to be a good candidate due to his penile prosthesis, which is a contraindication for water vapor thermal therapy. Instead, he underwent photoselective vaporization of the prostate (PVP) using a standard 26-Fr laser sheath with some notable resistance to scope manipulation. Upon removal of the sheath at the end of the case, implant tubing was seen in the urethra (Figure 1). The patient underwent successful open urethral repair the following day and was able to keep his IPP and it remains functional.

\section{Case 2: infection of penile prosthesis after suprapubic tube insertion}

A 75-year-old man who previously underwent infrapubic three-piece penile prosthesis placement for ED after radical prostatectomy 8 years prior subsequently developed urinary retention secondary to Parkinson's disease. Intermittent catheterization became progressively more difficult due to diminished hand dexterity; therefore, he elected to proceed with suprapubic tube placement. On post-operative day 15 after suprapubic tube placement the patient presented with suprapubic pain, tenderness, and erythema at the insertion site. He was started on empiric antibiotics, and a urine culture obtained. His urine culture grew multiple organisms and his antibiotics were tailored. His pain progressed to the area overlying the tubing for his prosthetic device and he developed purulent drainage. Computed tomography (CT) scan of the pelvis was performed, demonstrating close proximity of the suprapubic tube the prosthetic tubing with a small fluid collection. Given the findings, the patient underwent explant of his penile prosthesis. Culture of the device grew the same organisms as the urine culture. 


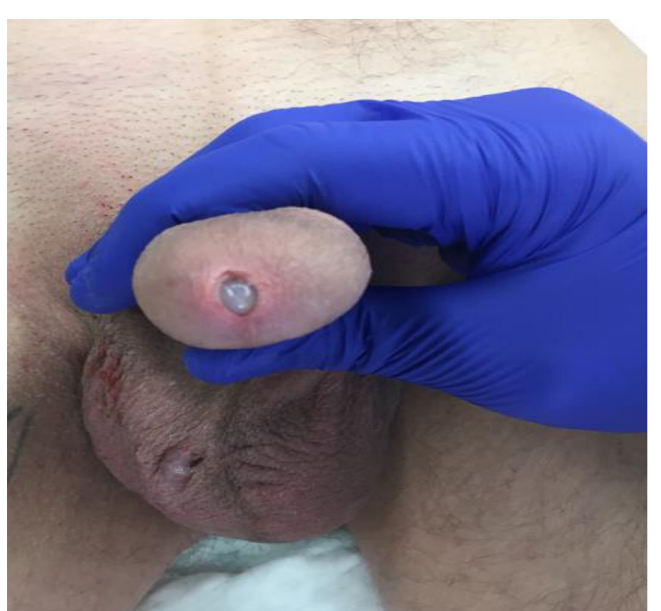

Figure 2 Extrusion of right cylinder following traumatic catheterization.

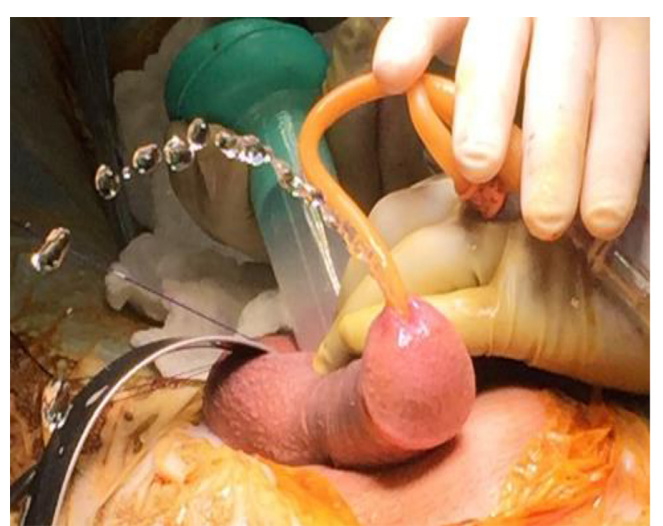

Figure 3 Intra operative irrigation revealing corpora-urethral fistula.

\section{Case 3: infection of penile prosthesis after traumatic catheter insertion}

A 64-year-old man who underwent insertion of a threepiece IPP 3 years prior presented to an outside hospital with altered mental status and acute kidney failure. He described initial attempt at catheter placement as painful and traumatic, traumatic with associated blood at the urethral meatus. Catheter was ultimately placed in the bladder with no resultant hematuria, and left in place for 10 days. Patient was discharged on hospital day 12 . He then presented to our facility 14 days later with a leukocytosis of 16 , in the setting of worsening left lower quadrant and penile pain. He underwent a CT scan of the abdomen and pelvis demonstrating a large fluid collection around his reservoir on the left side in the space of Retzius. Patient was started on broad spectrum intravenous antibiotics and taken to the operating room for explant of his device. Intraoperative findings included copious purulent fluid, which was cultured and grew Escherichia coli. Cystoscopy performed at the time of removal of his device did not reveal any sign of urethral injury.

\section{Case 4: extrusion of penile prosthesis after traumatic catbeter insertion}

A 57-year-old underwent with a history of meatal stenosis successfully underwent placement of a three-piece penile implant with standard use of a $16-\mathrm{Fr}$ catheter at the time. About 1 year post-operatively the patient presented to the emergency room with progressively decreasing stream. Urinalysis was normal but post-void residual (PVR) high. Catheter placement in the emergency room was noted to be extremely painful and resulted in immediate blood per meatus. The patient described the catheter was forcefully placed and resulted in a pop inward. The patient presented to our clinic a few days later with a cylinder extruding through the meatus (Figure 2). The patient underwent successful removal of the offending cylinder and utilized the device with one cylinder for 1 year. Irrigation of the corpora reveals the obvious corpora-urethral defect (Figure 3). The entire device was replaced for improved penile rigidity 1 year after the single cylinder removal.

\section{Case 5: bladder neck contracture at the time of PPS}

A 65-year-old gentleman presented for consideration of IPP for ED refractory to medical therapy secondary to robotic radical prostatectomy and adjuvant intensity-modulated radiation therapy for Gleason 4+5, pT4aN0Mx prostate adenocarcinoma treated at an outside institution 3 years prior. Despite denying LUTS on initial clinic visit, he subsequently underwent a PVR during his preoperative visit as is part of our standard workup. At that visit he voided $167 \mathrm{~mL}$, with a PVR of $260 \mathrm{~mL}$. On further questioning he admitted to having a slow urinary stream with delayed emptying. Preoperatively he was counseled that if there was any difficulty with catheter insertion, we would perform cystourethroscopy to rule out bladder neck contracture. At the time of surgery there was resistance with attempted insertion of a 14-Fr catheter, therefore cystourethroscopy was performed revealing a 4-Fr bladder neck contracture (Figure 4). A wire was threaded through the narrowing, 


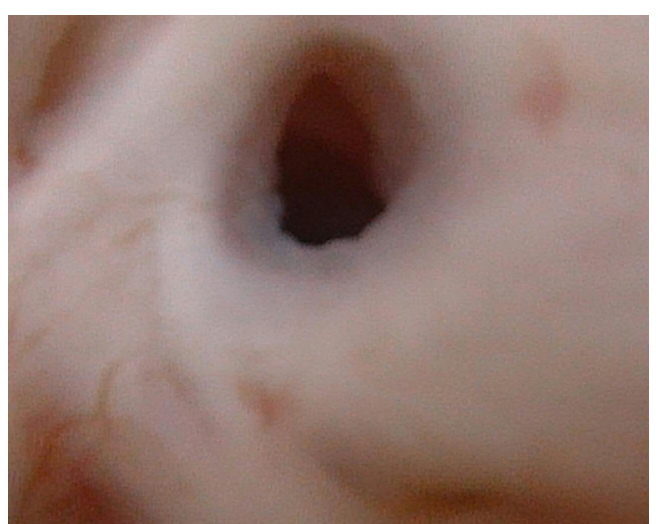

Figure 4 Cystoscopic view of bladder neck contracture in postprostatectomy patient.

dilated to 18 Fr using Goodwin sounds, and a 16-Fr catheter was placed for 48 hours. Upon removal of the catheter, he reported dramatic improvement in his voiding symptoms. At the time of publication our plan is to perform cystourethroscopy in 3 months and if he has developed a recurrent bladder neck contracture refer him to one of our colleagues for robotic bladder neck reconstruction.

\section{Methods}

A literature review was performed on PubMed using a combination of search terms including "penile prosthesis", "penile implant", "inflatable penile prosthesis", "benign prostatic hyperplasia", and "urinary retention." Only publications in English were considered. Apart from historical publications for context, emphasis was placed on newer literature distributed between 2000-2020.

\section{BPH work up in ED patients}

Given the prevalence of LUTS among ED patients, it is prudent to screen for $\mathrm{BPH}$ in men presenting for evaluation of sexual dysfunction. Per the most recent American Urological Association and European Association of Urology guideline statements, providers should obtain a relevant medical history, focused physical exam, urine analysis, and subjective assessment of symptoms using the American Urological Association Symptom Index or International Prostate Symptoms Score at time of initial evaluation $(12,13)$. Prior to surgical intervention for bladder outlet obstruction, patients should be further evaluated with a PVR, uroflowmetry, and prostate sizing with imaging or cystoscopy. Patients should be counseled regarding the effect of the various medical and surgical treatment options on erectile and ejaculatory function, as greater than $90 \%$ of patients considering BPH surgery report erectile and ejaculatory function to be an important consideration (14). For patients who desire preservation of their erectile and ejaculatory function, prostatic urethral lift (Urolift ${ }^{\circledR}$, Neo'Tract, Inc., Pleasanton, CA, USA), water vapor thermal therapy (Rezūm ${ }^{\mathrm{TM}}$, Boston Scientific, Marlborough, MA, USA), or Aquablation ${ }^{\circledR}$ (AquaBeam ${ }^{\circledR}$, PROCEPT BioRobotics Inc., Redwood Shores, CA, USA) may be better surgical options. Even newer surgical therapies still undergoing investigation that promote preservation of sexual function include a temporary nitinol implantable device (iTIND Olympus, Hamburg, Germany) and a BPH prostatic drug coated balloon dilation catheter (Optilume $^{\mathrm{TM}}$, Urotronic Inc., Plymouth, MN, USA). Patients with a history of prior surgical management for $\mathrm{BPH}$ should also be screened for LUTS prior to PPS. Judicious workup can help identify the isolated post-treatment patient who has been inadequately treated, has prostatic regrowth with new obstruction, or who has a urethral stricture from prior instrumentation. At a minimum, consider urine analysis and PVR in patients reporting residual or new LUTS after $\mathrm{BPH}$ surgery. Cystoscopy may be indicated to definitively diagnose a urethral stricture and/or prostatic regrowth, at which point focus should be re-directed to these conditions before PPS.

In patients with a history of radical prostatectomy or radiation therapy for prostate cancer, providers should consider the possibility of a bladder neck contracture. According to the CaPSURE database, at a mean followup of 2.7 years, $8.4 \%$ of radical prostatectomy patients, and $1.7 \%$ of external beam radiation therapy patients will develop a bladder neck contracture (15). Contemporary series that predominantly include robotic-assisted radical prostatectomy from large volume centers report bladder neck contracture rates of $1-2.5 \%$ (16-20). Prosthetic surgeons should be aware of this potential complication, as not all patients with a bladder neck contracture may complain of LUTS. An asymptomatic bladder neck contracture may not manifest until attempting to place an indwelling catheter for identification of the urethra at the time of PPS. This presents an avoidable conundrum intraoperatively if steps are taken preoperatively to rule out a bladder neck contracture. Some prosthetic surgeons perform cystoscopy or catheterization of all patients with a history of treatment for prostate cancer preoperatively, 
while others utilize it in selective cases. Some implant surgeons will abort all implant procedures if bladder neck contracture is present, while others will proceed only if dilation goes smoothly despite the theoretically increased risk of infection. An additional potential problem is that stress incontinence may be revealed with treatment of bladder neck contracture. Treatment of stress urinary incontinence should ideally occur prior to placement of penile prosthesis.

\section{Treatment of BPH prior to PPS}

While there are no formal guidelines addressing the optimal timing and management of $\mathrm{BPH}$ in patients undergoing PPS, most providers agree that treating $\mathrm{BPH}$ before PPS is prudent to prevent device infection and/ or erosion. According to the Titan and Titan Touch ${ }^{\circledR}$ IPP product insert (Coloplast Corp., Minneapolis, MN, USA), an elevated residual urine volume secondary to bladder outlet obstruction or neurogenic bladder is a contraindication for use of the device (21). Aside from the negative effect on quality of life, severe LUTS left untreated can be complicated by acute urinary retention (AUR), recurrent urinary tract infections, bladder stones, and renal failure (22). PPS should be deferred indefinitely until these have been ruled out and addressed. In patients with mild to moderate LUTS without the aforementioned conditions, surgical BPH treatment should be explored prior to PPS given that transurethral intervention after cylinder placement may be more difficult. Providers should be cognizant of the pressure exerted by a rigid cystoscope or resectoscope on the urethra, and potential for trauma to the corpus spongiosum in close proximity to the prosthetic device.

Patients with BPH with or without LUTS and a history of AUR are at risk of subsequent retention episodes (23). Postoperative urinary retention is a common sequela after any type of surgery, with an overall reported incidence of $4-29 \%$ (24). Common risk factors include preexisting bladder outlet obstruction, prior AUR episodes, advanced age, diabetes mellitus, type of surgery and prolonged anesthesia, but can also occur in younger patients or those without prior episodes (25-28). One potential mechanism for AUR attributable to these factors includes temporary or permanent loss of bladder afferent and/or efferent pathways.

The risk of postoperative AUR after PPS is approximately $4 \%$ (29). In general, AUR patients can be managed with either indwelling urinary catheter or clean intermittent catheterization (CIC) for a short duration of time until spontaneous symptom resolution. In a postPPS patient, CIC is preferred to avoid prolonged pressure exerted by an indwelling catheter in the setting of a prosthetic device to minimize the risk of erosion. Even in patients without AUR, chronically elevated PVR secondary to untreated $\mathrm{BPH}$ contributes to urine stagnation; placing the patient at an increased risk of a urinary and potentially device infection. Taken together, in a patient with known risk factors for postoperative AUR undergoing surgery that places them at increased risk of catheter associated complications, it is prudent to address bladder outlet obstruction before proceeding with device implantation.

There is an ever-increasing array of treatment options for $\mathrm{BPH}$, with transurethral resection of the prostate (TURP) being the gold standard. With this classic approach, it is important to counsel the patient on the complications which directly impact sexual health. These would include retrograde ejaculation (23-65\%), de novo ED $(6 \%)$, and urethral stricture disease $(4 \%)(12,30)$. Alternatives to TURP including transurethral incision of the prostate (TUIP), holmium laser enucleation of the prostate (HOLEP), and PVP all have an increased risk of ED and retrograde ejaculation. Prior to 2015, the only BPH surgical treatment that didn't pose a risk of ED or retrograde ejaculation was prostatic urethral lift. In recent years, water vapor thermal therapy has shown promise in terms of its minimally invasive nature as well as preservation of erectile and ejaculatory function at 36-month followup, however, it is contraindicated in patients with a penile prosthesis for reasons that are unclear, as it does not seem to pose a greater risk than any other BPH surgery (12). A newer still treatment modality known as Aquablation ${ }^{\circledR}$ (Procept Biorobotics, Redwood Shores, CA, USA) also does not appear to increase the risk of ED, but can still cause retrograde ejaculation albeit at much reduced rate when compared to TURP (31-39). Understanding the risks and benefits of the available approaches can help guide patients to the best modality based on their individual preferences and prostate anatomy. Following $\mathrm{BPH}$ surgery, it is our practice to wait at least 8 weeks for resolution of irritative voiding symptoms and hematuria prior to proceeding with PPS. Failure to do so may delay diagnosis of a urinary tract infection or urethral injury in the perioperative period, as well as increase the risk of postoperative AUR.

\section{Treatment of BPH after PPS}

While there are clear advantages to treating $\mathrm{BPH}$ prior 
to IPP placement, this is not always possible. Patients may present with symptomatic BPH after having undergone PPS elsewhere, with symptomatic regrowth after being appropriately treated prior to PPS, and with de novo symptoms after having PPS years prior. In all the aforementioned scenarios, a thorough discussion of management options, risks, and benefits should be explored with the patient. A trial of medical management should be considered in treatment-naive patients. For those who meet clinical indications for surgical BPH treatment (i.e., recurrent urinary tract infections, bladder stones, urinary retention, and recurrent gross hematuria), the quickest and least invasive route should be employed when possible. The minimally invasive surgical therapies water vapor thermal therapy and prostatic urethral lift can both be performed using a 20-Fr rigid cystoscope sheath, Aquablation a 22-Fr sheath, Greenlight ${ }^{\mathrm{TM}}$ Laser PVP (Boston Scientific, Boston Scientific, Marlborough, MA, USA) a 26-Fr sheath, TURP a 24- or 26-Fr sheath and HOLEP a 24- to 28-Fr sheath. Simple prostatectomy may be less desirable due to the need for an indwelling catheter in the postoperative period, as well as the potential for contamination or damage to the reservoir. In addition to invasiveness of procedure, prostate size and surgeon expertise should drive selection of $\mathrm{BPH}$ surgery type. There is a paucity of literature for surgical management of $\mathrm{BPH}$ after PPS, with no literature reports of prosthesis infection after BPH surgery. Anecdotally, the authors have observed prosthesis infection after water vapor thermal therapy and PVP. While the corporal bodies diverge proximally, prostatic capsular perforation does expose this usually sterile space to urine extravasation, increasing the risk of an infection.

\section{Management of urinary retention in post-PPS}

With the infrapubic and penoscrotal approach in PPS, a urinary catheter is typically inserted at time of surgery to aid in identification of the urethra during dissection and allows for decompression of the bladder in anticipation for safe reservoir placement. The catheter is then removed prior to discharge same day, or early postoperative day 1 for patients admitted overnight. Literature reports of postoperative AUR after PPS range between 1-4\% (29,40-43). Prolonged use of an indwelling urinary catheter is discouraged in PPS patients given concerns for urethral erosion leading to prosthesis infection. There are reports of cylinder erosion into the urethra in patients with chronic catheter pressure (44). This is most commonly seen in patients with semi rigid rods and decreased penile sensation, such as those with spinal cord injuries $(45,46)$. Data from the artificial urinary sphincter literature demonstrates that prolonged catheterization (greater than 48 hours) is reported to increase the risk of device erosion, while data is sparse on the potential impact of self-catheterization in a non-neurogenic adult population $(47,48)$. Whether these findings can be extrapolated to PPS is debatable, but most prosthetic surgeons would rather err on the side of caution rather than risk a prosthetic infection.

Management of urethral erosion in the penile prosthesis patient can prove difficult depending on the site of injury, extent of injury, and patient factors that affect wound healing. A prosthetic device in which any component is visible externally is by historical definition infected. Erosion will disseminate microorganisms throughout the previously sterile implant space necessitating explantation of the cylinders and/or entire device. The magnitude of this complication is likely underreported as it is limited to case reports as it is often a delayed complication presenting years later, often to a different provider. Though uncommon, the ramifications of this particular complication have steered providers away from prolonged use of an indwelling catheter after PPS. A more attractive alternative is CIC, which has been shown to be safe in previous studies (49). In a small prospective trial of men undergoing elective inguinal herniorrhaphy surgery, preoperative tamsulosin administration have been shown to reduce the incidence of postoperative AUR, though is not commonly done in practice (50). A less common scenario is the patient with chronic postoperative urinary retention who is not a candidate for BPH surgery and cannot perform CIC, in which case a suprapubic tube may be a more attractive alternative. Even suprapubic tube insertion poses a risk for device infection; the authors have explanted an IPP placed through an infrapubic incision where the reservoir tubing was in close contact to the suprapubic tube insertion site and subsequently became infected.

\section{Intraoperative management of difficult catheter placement}

High volume prosthetic surgeons will undoubtedly encounter difficulty inserting a catheter at the time of PPS on occasion. Potential causes of such resistance include a urethral stricture, bladder neck contracture, and prostatic obstruction. Most prosthetic surgeons will place a 14- or 16-Fr catheter at the time of PPS to aid in identification 
during dissection near the urethra. In our practice we use a 14-Fr coude catheter (16-Fr straight tip catheter in radical prostatectomy patients) under the assumption that a significant percentage of PPS patients benefit from the curved tip of the coude catheter to navigate the prostatic urethra. We elect to use a 14-Fr to reduce the compressive effect on the urethra to minimize the risk of glans ischemia.

In the case where a catheter will not pass, options include omitting catheter placement versus using a flexible cystoscope to identify the point of obstruction. In the absence of a catheter, the reservoir should be placed in an ectopic position to avoid injury to a bladder that is not decompressed. Another consideration in this situation is how to manage post-operative AUR should it arise. Decompression of the bladder will require instrumentation with a prosthetic device in place, which is undesirable. Alternatively, addressing the obstruction at the time of PPS may require urethral dilation, direct vision internal urethrotomy, complex catheter placement over a guidewire to navigate a false passage, or bladder neck dilation/incision. Any of these maneuvers may violate the surrounding corpus spongiosum and/or corpus cavernosum thereby increasing the risk of infection. Depending on the point of obstruction, the patient may need to be repositioned into the lithotomy position.

It should also be noted that given the unanticipated findings, consent will not have been obtained for this intervention, as a discussion regarding this scenario is unlikely to have taken place preoperatively. Caution should be exercised in patients with a urethral stricture near the external urethral sphincter or at the bladder neck in postprostatectomy patients, especially with a history of radiation therapy because intervention may convert a previously continent patient into one with stress incontinence. The decision to proceed with PPS as planned versus addressing the obstruction and aborting the prosthesis portion of the case should be made carefully. It is unlikely that the prosthetic surgeon will regret aborting a case only to return at a later date, but may wish that he or she had chosen to abort the case when faced with urethral bleeding, hematuria, or the prospect of a prolonged indwelling catheter in the postoperative setting. In situations where urethral or bladder neck dilation/incision is performed, the authors would advocate waiting a minimum of three months, repeating a cystoscopy, and reassessing whether to proceed with PPS.

Another infrequent scenario that may arise is an elevated PVR at the time of catheter placement intraoperatively.
To avoid this unanticipated event, we obtain a PVR on all patients scheduled for PPS ahead of time. Occasionally patients will have an elevated PVR, prompting further discussion regarding their urinary habits and symptoms. Patients with a mildly elevated PVR may benefit from initiation of an alpha-blocker preoperatively, while those with a significantly elevated PVR should undergo further workup to rule out obstructive BPH. A preoperative PVR is valuable in diagnosing asymptomatic urinary retention and interpreting elevated PVRs in the postoperative period.

\section{Discussion}

$\mathrm{BPH}$ and ED are common comorbid conditions that should be on the radar of prosthetic surgeons during the initial workup process. A conservative surgical approach would dictate treating LUTS due to BPH prior to PPS. This ensures the patient's urinary symptoms have been adequately addressed, and mitigates any potentially avoidable complications of BPH surgery after PPS. Providers should allow an adequate recovery period to achieve symptomatic relief and navigate manifestations of the most common complications of $\mathrm{BPH}$ surgery in the postoperative period. In instances where BPH surgery must be performed in a patient with an existing implant, providers should consider the least invasive surgical technique within their armamentarium. In patients with AUR after PPS, CIC is preferred over indwelling catheter to minimize the risk of urethral erosion and device infection.

The above work is limited by paucity of high-quality literature on BPH in the PPS population. Additional work is needed in the field to better characterize $\mathrm{BPH}$ in the prosthetic urology setting and delineate the optimal $\mathrm{BPH}$ treatment modality following PPS surgery. As advancements in $\mathrm{BPH}$ treatment introduces an ever-growing array of managements options, patients will need guidance on the order and manner of proceeding should they also wish to undergo PPS. Understanding these above basic treatment principles can help direct better management of ED patients undergoing consideration for penile implantation.

\section{Acknowledgments}

Funding: None.

\section{Footnote}

Provenance and Peer Review: This article was commissioned 
by the editorial office, Translational Andrology and Urology for the series "Controversies and Considerations of Penile Surgery". The article has undergone external peer review.

Reporting Checklist: The authors have completed the Narrative Review Reporting Checklist. Available at: http:// dx.doi.org/10.21037/tau-20-1225.

Peer Review File: Available at http://dx.doi.org/10.21037/ tau-20-1225

Conflicts of Interest: The authors have completed the ICMJE uniform disclosure form (available at http://dx.doi. org/10.21037/tau-20-1225). The series "Controversies and Considerations of Penile Surgery" was commissioned by the editorial office without any funding or sponsorship. TSK served as the unpaid Guest Editor of the series and serves as an unpaid Associate Editor-in-Chief of Translational Andrology and Urology from Jan 2020 to Dec 2021. The authors have no other conflicts of interest to declare.

Ethical Statement: The authors are accountable for all aspects of the work in ensuring that questions related to the accuracy or integrity of any part of the work are appropriately investigated and resolved.

Open Access Statement: This is an Open Access article distributed in accordance with the Creative Commons Attribution-NonCommercial-NoDerivs 4.0 International License (CC BY-NC-ND 4.0), which permits the noncommercial replication and distribution of the article with the strict proviso that no changes or edits are made and the original work is properly cited (including links to both the formal publication through the relevant DOI and the license). See: https://creativecommons.org/licenses/by-nc-nd/4.0/.

\section{References}

1. Montorsi F, Rigatti P, Carmignani G, et al. AMS threepiece inflatable implants for erectile dysfunction: a longterm multi-institutional study in 200 consecutive patients. Eur Urol 2000;37:50-5.

2. Li K, Brandes ER, Chang SL, et al. Trends in penile prosthesis implantation and analysis of predictive factors for removal. World J Urol 2019;37:639-46.

3. Mirheydar HS, Palazzi KL, Parsons JK, et al. Hospitalbased trends in penile prosthetic surgery. J Sex Med 2015;12:1092-8.
4. Braun MH, Sommer F, Haupt G, et al. Lower urinary tract symptoms and erectile dysfunction: co-morbidity or typical "Aging Male" symptoms? Results of the "Cologne Male Survey". Eur Urol 2003;44:588-94.

5. Hoesl CE, Woll EM, Burkart M, et al. Erectile dysfunction (ED) is prevalent, bothersome and underdiagnosed in patients consulting urologists for benign prostatic syndrome (BPS). Eur Urol 2005;47:511-7.

6. Rosen R, Altwein J, Boyle P, et al. Lower urinary tract symptoms and male sexual dysfunction: the multinational survey of the aging male (MSAM-7). Eur Urol 2003;44:637-49.

7. Gonzalez RR, Kaplan SA. Tadalafil for the treatment of lower urinary tract symptoms in men with benign prostatic hyperplasia. Expert Opin Drug Metab Toxicol 2006;2:609-17.

8. Chung WS, Nehra A, Jacobson DJ, et al. Lower urinary tract symptoms and sexual dysfunction in communitydwelling men. Mayo Clin Proc 2004;79:745-9.

9. Boyle P, Robertson C, Mazzetta C, et al. The association between lower urinary tract symptoms and erectile dysfunction in four centres: the UrEpik study. BJU Int 2003;92:719-25.

10. Ponholzer A, Temml C, Obermayr R, et al. Association between lower urinary tract symptoms and erectile dysfunction. Urology 2004;64:772-6.

11. De Nunzio C, Roehrborn CG, Andersson KE, et al. Erectile Dysfunction and Lower Urinary Tract Symptoms. Eur Urol Focus 2017;3:352-63.

12. Foster HE, Barry MJ, Dahm P, et al. Surgical Management of Lower Urinary Tract Symptoms Attributed to Benign Prostatic Hyperplasia: AUA Guideline. J Urol 2018;200:612-19.

13. Parsons JK, Dahm P, Köhler TS, et al. Surgical Management of Lower Urinary Tract Symptoms Attributed to Benign Prostatic Hyperplasia: AUA Guideline Amendment 2020. J Urol 2020;204:799-804.

14. Bouhadana D, Nguyen DD, Zorn KC, et al. Patient Perspectives on Benign Prostatic Hyperplasia Surgery: A Focus on Sexual Health. J Sex Med 2020;17:2108-12.

15. Elliott SP, Meng MV, Elkin EP, et al. Incidence of urethral stricture after primary treatment for prostate cancer: data From CaPSURE. J Urol 2007;178:529-34; discussion 534.

16. Breyer BN, Davis CB, Cowan JE, et al. Incidence of bladder neck contracture after robot-assisted laparoscopic and open radical prostatectomy. BJU Int 2010;106:1734-8.

17. Hamdy FC, Donovan JL, Lane JA, et al. 10-Year Outcomes after Monitoring, Surgery, or Radiotherapy 
for Localized Prostate Cancer. N Engl J Med 2016;375:1415-24.

18. Erickson BA, Meeks JJ, Roehl KA, et al. Bladder neck contracture after retropubic radical prostatectomy: incidence and risk factors from a large single-surgeon experience. BJU Int 2009;104:1615-9.

19. Giudice CR, D'Alessandro FJ, Galarza GA, et al. Surgical approach to vesicourethral anastomotic stricture following radical prostatectomy. Actas Urol Esp 2016;40:124-30.

20. Parihar JS, Ha YS, Kim IY. Bladder neck contractureincidence and management following contemporary robot assisted radical prostatectomy technique. Prostate Int 2014;2:12-8.

21. Titan Touch Product Information \& Resources. Available online: https://www.coloplast.us/titan-touchen-us.aspx\#section=product-description_3. Accessed 08/21/20202020.

22. O'Leary MP. Lower urinary tract symptoms/benign prostatic hyperplasia: maintaining symptom control and reducing complications. Urology 2003;62:15-23.

23. Roehrborn CG, Bruskewitz R, Nickel GC, et al. Urinary retention in patients with $\mathrm{BPH}$ treated with finasteride or placebo over 4 years. Characterization of patients and ultimate outcomes. The PLESS Study Group. Eur Urol 2000;37:528-36.

24. Darrah DM, Griebling TL, Silverstein JH. Postoperative urinary retention. Anesthesiol Clin 2009;27:465-84, table of contents.

25. Tammela T, Kontturi M, Lukkarinen O. Postoperative urinary retention. I. Incidence and predisposing factors. Scand J Urol Nephrol 1986;20:197-201.

26. Sarasin SM, Walton MJ, Singh HP, et al. Can a urinary tract symptom score predict the development of postoperative urinary retention in patients undergoing lower limb arthroplasty under spinal anaesthesia? A prospective study. Ann R Coll Surg Engl 2006;88:394-8.

27. Liang CC, Lee CL, Chang TC, et al. Postoperative urinary outcomes in catheterized and non-catheterized patients undergoing laparoscopic-assisted vaginal hysterectomy--a randomized controlled trial. Int Urogynecol J Pelvic Floor Dysfunct 2009;20:295-300.

28. Ringdal M, Borg B, Hellstrom AL. A survey on incidence and factors that may influence first postoperative urination. Urol Nurs 2003;23:341-6, 354.

29. Gupta NK, Ring J, Trost L, et al. The penoscrotal surgical approach for inflatable penile prosthesis placement. Transl Androl Urol 2017;6:628-38.

30. Madersbacher S, Marberger M. Is transurethral resection of the prostate still justified? BJU Int 1999;83:227-37.

31. Bhojani N, Nguyen DD, Kaufman RP Jr, et al. Comparison of $<100 \mathrm{cc}$ prostates and $>100 \mathrm{cc}$ prostates undergoing aquablation for benign prostatic hyperplasia. World J Urol 2019;37:1361-8.

32. Chughtai B, Thomas D. Pooled Aquablation Results for American Men with Lower Urinary Tract Symptoms due to Benign Prostatic Hyperplasia in Large Prostates (60-150 cc). Adv Ther 2018;35:832-8.

33. Desai MM, Singh A, Abhishek S, et al. Aquablation therapy for symptomatic benign prostatic hyperplasia: a single-centre experience in 47 patients. BJU Int 2018;121:945-51.

34. Gilling P, Anderson P, Tan A. Aquablation of the Prostate for Symptomatic Benign Prostatic Hyperplasia: 1-Year Results. J Urol 2017;197:1565-72.

35. Gilling P, Barber N, Bidair M, et al. WATER: A DoubleBlind, Randomized, Controlled Trial of Aquablation((R)) vs Transurethral Resection of the Prostate in Benign Prostatic Hyperplasia. J Urol 2018;199:1252-61.

36. Gilling P, Reuther R, Kahokehr A, et al. Aquablation - image-guided robot-assisted waterjet ablation of the prostate: initial clinical experience. BJU Int 2016;117:923-9.

37. Kasivisvanathan V, Hussain M. Aquablation versus transurethral resection of the prostate: 1 year United States - cohort outcomes. Can J Urol 2018;25:9317-22.

38. Yafi FA, Tallman CT, Seard ML, et al. Aquablation outcomes for the U.S. cohort of men with LUTS due to $\mathrm{BPH}$ in large prostates $(80-150 \mathrm{cc})$. Int J Impot Res 2018;30:209-14.

39. Zorn KC, Goldenberg SL, Paterson R, et al. Aquablation among novice users in Canada: A WATER II subpopulation analysis. Can Urol Assoc J 2019;13:E113-8.

40. Kessler R. Complications of inflatable penile prostheses. Urology 1981;18:470-2.

41. Minervini A, Ralph DJ, Pryor JP. Outcome of penile prosthesis implantation for treating erectile dysfunction: experience with 504 procedures. BJU Int 2006;97:129-33.

42. Garber BB. Outpatient inflatable penile prosthesis insertion. Urology 1997;49:600-3.

43. Mulhall JP, Bloom K. Comparison of in-patient and out-patient penile prosthesis surgery. Int J Impot Res 2001;13:251-4.

44. Steidle CP, Mulcahy JJ. Erosion of penile prostheses: a complication of urethral catheterization. J Urol 1989;142:736-9.

45. Hisasue S, Sato Y, Horita H, et al. Erosion of a penile 
prosthesis due to an indwelling urethral catheter as a late complication. Int J Urol 2002;9:525-7.

46. Zermann DH, Kutzenberger J, Sauerwein D, et al. Penile prosthetic surgery in neurologically impaired patients: long-term followup. J Urol 2006;175:1041-4; discussion 1044.

47. Martins FE, Boyd SD. Post-operative risk factors associated with artificial urinary sphincter infectionerosion. Br J Urol 1995;75:354-8.

Cite this article as: Wen L, Köhler TS, Helo S. A narrative review of the management of benign prostatic hyperplasia in patients undergoing penile prosthesis surgery. Transl Androl Urol 2021;10(6):2695-2704. doi: 10.21037/tau-20-1225
48. Seideman CA, Zhao LC, Hudak SJ, et al. Is prolonged catheterization a risk factor for artificial urinary sphincter cuff erosion? Urology 2013;82:943-6.

49. Diokno AC, Sonda LP. Compatibility of genitourinary prostheses and intermittent self-catheterization. J Urol 1981;125:659-60.

50. Mohammadi-Fallah M, Hamedanchi S, Tayyebi-Azar A. Preventive effect of tamsulosin on postoperative urinary retention. Korean J Urol 2012;53:419-23. 